\title{
BOUNDS ON THE NORM OF A SOLUTION OF A GENERAL DIFFERENTIAL EQUATION
}

\author{
C. E. LANGENHOP ${ }^{1}$
}

1. In this note we establish upper and lower bounds on the norm of a solution of the equation

$$
\frac{d z}{d x}=F(x, z) .
$$

Upper bounds obtained by application of Bellman's lemma [1, p. 35] and its generalization by Bihari [2] have been much used in the study of solutions of equations such as (1.1). Similar methods permit the determination of analogous lower bounds which seem to be unknown until now. In $\$ 2$ we present a result which might indicate why this is the case. Concerning (1.1) we make the following assumptions:

(1) $x$ is a real variable, $z$ and $F$ are finite dimensional complex vectors with $n$ components $z_{i}$ and $F_{i}$ respectively,

(2) $F$ is continuous in $(x, z)$ for all $z$ and all $x \in[a, b]$, i.e. $a \leqq x \leqq b$ with $a<b$,

(3) for some norm, say $|z|=\sum_{i=1}^{n}\left|z_{i}\right|, F$ satisfies

$$
|F(x, z)| \leqq v(x) g(|z|)
$$

where

(4) $v(x)$ is continuous and $v(x) \geqq 0$ for $x \in[a, b]$ and

(5) $g(u)$ is continuous and nondecreasing for $u \geqq 0$ and $g(u)>0$ for $u>0$.

TheORem 1. Let $z(x)$ be continuous, satisfy $|z(x)|>0$ and be $a$ solution of (1.1) for $x \in[a, b]$, where $F$ satisfies conditions (1) through (5) above. Then for $x \in[a, b]$

$$
|z(x)| \leqq G^{-1}\left(G(|z(a)|)+\int_{a}^{x} v(s) d s\right)
$$

where

$$
G(u)=\int_{u_{0}}^{u}[g(t)]^{-1} d t, \quad u_{0} \geqq 0,
$$

and

Received by the editors September 29, 1959 and, in revised form, December 2, 1959.

1 This research was sponsored by the Office of Ordnance Research, U. S. Army. 


$$
|z(x)| \geqq G^{-1}\left(G(|z(a)|)-\int_{a}^{x} v(s) d s\right)
$$

for all $x \in[a, b]$ for which $G(|z(a)|)-\int_{a}^{x} v(s) d s$ is in the domain of $G^{-1}$.

Proof. We have $d z(x) / d x=F(x, z(x))$ so that

$$
z(x)=z(y)+\int_{y}^{x} F(s, z(s)) d s, \quad x, y \in[a, b] .
$$

Let $u(x)=|z(x)|$. Then from (1.5) and (1.2) we have for $y \leqq x$

$$
u(x) \leqq u(y)+\int_{y}^{x} v(s) g(u(s)) d s
$$

and

$$
u(x) \geqq u(y)-\int_{y}^{x} v(s) g(u(s)) d s .
$$

We shall prove that (1.4) follows from (1.7) and merely indicate how (1.3) follows from (1.6) since this latter is essentially Bihari's result.

For fixed $x$ in the interval $a<x \leqq b$ we define for $a \leqq y \leqq x$

$$
\phi(y)=u(x)+\int_{y}^{x} v(s) g(u(s)) d s .
$$

Then $\phi^{\prime}(y)=-v(y) g(u(y))$ so by (1.7) and conditions (4) and (5) it follows that

$$
\phi^{\prime}(y)+v(y) g(\phi(y)) \geqq 0 .
$$

Since $\phi(y)>0$, condition (5) and the definition of $G$ along with (1.9) imply that

$$
\frac{d}{d y} G(\phi(y))+v(y) \geqq 0,
$$

integration of which leads to

$$
G(\phi(x))-G(\phi(y))+\int_{y}^{x} v(s) d s \geqq 0, \quad a \leqq y \leqq x \leqq b .
$$

By (1.8) we have $\phi(x)=u(x)$ so (1.11), (1.7) and the monotonicity of $G$ imply that

$$
G(u(x)) \geqq G(u(y))-\int_{y}^{x} v(s) d s .
$$


Setting $y=a$ and using the monotonicity of $G^{-1}$, we are led to (1.4) for those $x \in[a, b]$ for which $G(u(a))-\int_{a}^{x} v(s) d s$ is in the domain of $G^{-1}$.

The proof of (1.3) may be carried out similarly except we may immediately set $y=a$ in (1.6) and define $\psi(x)=u(a)+\int_{a}^{x} v(s) g(u(s)) d s$. Then $\psi^{\prime}(x)=v(x) g(u(x))$ and the remaining details are analogous to the above.

2. Inequality (1.3) and particularly its specialization in the case $g(u) \equiv u$ (Bellman's lemma) have been used extensively in the theory of differential equations. That its counterpart (1.4) has been unknown up to now is perhaps due to the fact that it can not be proven in the same way as was just indicated for (1.3). That is to say, if we set $y=a$ in (1.7), then the result, even though it holds for all $x \in[a, b]$, does not imply (1.4). We shall show this for the special case $g(u) \equiv u$. This may be stated as follows. Let $u(x)$ and $v(x)$ be continuous on the interval $[a, b]$ and let $u(x) \geqq 0$ and $v(x) \geqq 0$ on this interval but $v(x) \not \equiv 0$. Then the condition

$$
u(x) \geqq u(a)-\int_{a}^{x} v(s) u(s) d s
$$

does not imply

$$
u(x) \geqq u(a) \exp \left[-\int_{a}^{x} v(s) d s\right] .
$$

We shall prove the equivalent

THEOREM 2. Let $v(x)$ be continuous and such that $v(x) \geqq 0$ but $v(x) \not \equiv 0$ on $[a, b]$ where $a<b$. Then there exist continuous functions $u(x), w(x)$ which are non-negative on $[a, b]$ and an $x_{0} \in[a, b]$ such that

$$
u(x)=u(a)-\int_{a}^{x} v(s) u(s) d s+w(x)
$$

on $[a, b]$ but

$$
u\left(x_{0}\right)<u(a) E\left(x_{0}, a\right)
$$

where

$$
E(x, y)=\exp \left[\int_{x}^{y} v(s) d s\right] .
$$

REMARK. If one does not insist that $u(x)$ be non-negative the result 
is much more easily established, but the inequality (2.1) arises naturally in differential equation theory and in this case $u(x) \geqq 0$.

Proof of Theorem 2. From the hypotheses regarding $v$ it follows that there is an interval $\left[a_{1}, b_{1}\right]$ such that $a_{1}<b_{1}$ and

$$
v(x)>0, \quad a_{1} \leqq x \leqq b_{1} .
$$

Consider the function $h$ defined by

$$
h(x)=\int_{a_{1}}^{x} E\left(s, b_{1}\right) d s-\int_{x}^{b_{1}} E\left(s, b_{1}\right) d s .
$$

Clearly $h(x)$ is continuous and $h\left(a_{1}\right)<0$. On the other hand it follows from (2.5) that $h\left(\left(a_{1}+b_{1}\right) / 2\right)>0$. Hence there exists an $x_{1}$, such that

$$
h\left(x_{1}\right)=0, \quad a_{1}<x_{1}<\left(a_{1}+b_{1}\right) / 2 .
$$

Having chosen $x_{1}$ as in (2.7) we select $u(a)$ such that

$$
u(a)>\left(a_{1}+b_{1}-2 x_{1}\right) E\left(a, b_{1}\right) .
$$

Obviously $u(a)>0$. Now we define a function $r$ by the equations

$$
r(x)=\left\{\begin{array}{lr}
0, & a \leqq x \leqq a_{1} \\
x-a_{1}, & a_{1} \leqq x \leqq x_{1} \\
2 x_{1}-a_{1}-x, & x_{1} \leqq x \leqq b_{1} \\
\left(2 x_{1}-a_{1}-b_{1}\right) E\left(x, b_{1}\right), & b_{1} \leqq x \leqq b .
\end{array}\right.
$$

The function $w(x)$ is now defined to be the solution of

$$
w(x)=r(x)+\int_{a}^{x} w(s) v(s) E(x, s) d s
$$

and in turn $u(x)$ is defined to be the corresponding solution of (2.3) with $u(a)$ chosen to satisfy (2.8).

It may be verified directly that $u(x)$ given by

$$
u(x)=u(a) E(x, a)+w(x)-\int_{a}^{x} w(s) v(s) E(x, s) d s
$$

satisfies (2.3), or conversely this solution may be obtained by solving the equation $(d / d x)[u(x)-w(x)]=-v(x) u(x)$ which arises from (2.3). From (2.10) and (2.11) it then follows that

$$
u(x)=u(a) E(x, a)+r(x)
$$

and since $r\left(b_{1}\right)<0$ the inequality (2.4) follows for $x_{0}=b_{1}$. 
It remains to show then that $w(x) \geqq 0$ and $u(x) \geqq 0$. The explicit form of the solution $w(x)$ of $(2.10)$ is

$$
w(x)=r(x)+\int_{a}^{x} r(s) v(s) E(s, x) d s
$$

which may be obtained in the same way as was indicated for (2.11). Since $r(x)$ is continuous we may rewrite (2.13), after integrating by parts, in the form of a Stieltjes integral

$$
w(x)=\int_{a}^{x} E(s, x) d r(s) .
$$

Consider now the function $w(x) E\left(x, b_{1}\right)=\int_{a}^{x} E\left(s, b_{1}\right) d r(s)$. It is clear from the form of $r(x)$ as given in (2.9) that this function is nondecreasing on the interval $\left[a, x_{1}\right]$, nonincreasing on the interval $\left[x_{1}, b_{1}\right]$ and again nondecreasing on the interval $\left[b_{1}, b\right]$. Hence the minimum value of this function is attained at either $x=a$ or $x=b_{1}$. But its value at $x=a$ is clearly zero while its value at $x=b_{1}$ is also zero by choice of $x_{1}$ (see (2.6) and (2.7)). Hence this function is non-negative on $[a, b]$ and so also then is $w(x)$.

Turning now to $u(x)$ we observe from (2.12) and (2.9) that certainly $u(x) \geqq 0$ on the interval $\left[a, x_{1}\right]$. On the interval $\left[x_{1}, b_{1}\right] r(x)$ is decreasing as is the term $u(a) E(x, a)$ so the minimum value of $u(x)$ on this interval is attained at $x=b_{1}$. Thus we need verify $u(x) \geqq 0$ only on the interval $\left[b_{1}, b\right]$. Here we have

$$
u(x)=\left[u(a)-\left(a_{1}+b_{1}-2 x_{1}\right) E\left(a, b_{1}\right)\right] E(x, a)
$$

which is clearly positive by (2.8). Hence $u(x) \geqq 0$ for all $x \in[a, b]$ and the theorem is proved.

\section{REFERENCES}

1. R. Bellman, Stability theory of differential equations, New York, McGraw-Hill Book Company, Inc., 1953.

2. I. Bihari, $A$ generalization of a lemma of Bellman and its application to uniqueness problems of differential equations, Acta Math. Acad. Sci. Hungar. vol. 7 (1956) pp. 81-94.

Iowa State University of Science and Technology 